\title{
An improved block diagonal precoding scheme for MIMO multicast channel with two users
}

\author{
Xiaodong Xu*, Zilong Zhang and Baisheng Du
}

\begin{abstract}
Matrix theory plays an important role in precoding methodology for multiple input multiple output (MIMO) systems. In this paper, an improved block diagonal (BD) precoding scheme is proposed for a MIMO multicast channel with two users, where the unitary precoding matrix is constructed in a block-wise form by joint triangularization decomposition. In order to reduce large signal-to-noise ratios (SNRs) spread across different transmitted data streams and users, the combination of joint equi-diagonal triangularization (JET) and joint geometric mean decomposition (JGMD) is applied to submatrix construction in the inner process of this precoding scheme. An elaborate implementation is presented, and the existence condition of JGMD is also investigated for two complex-valued matrices with two columns, where the analytical result reveals the connection with the particular channel realization and essentially determines when to consider JGMD for submatrix construction. In addition, the properties of the diagonal elements generated by joint triangularization decomposition are discussed as well as the computational complexity of the proposed scheme. Simulation results indicate that in general, JGMD is employed with high probability in the hybrid model, and the proposed scheme readily outperforms the JET scheme in terms of bit error rate (BER) performance in the moderate to high SNR regimes.
\end{abstract}

Keywords: MIMO; Multicast; Precoding; Block diagonal; JGMD

\section{Introduction}

Precoding techniques have gained extensive research interests in the literature due to their capability of achieving the ergodic capacity and improving link reliability for wireless communication over multiple input multiple output (MIMO) channels (see, e.g., [1] and the references therein). Under the assumption of full channel state information (CSI) at the transmit side as well as at the receive side, matrix theory, including several decomposition approaches, is often used as a basic tool to facilitate the precoder design and performance analysis for different types of communication scenarios. For instance, singular value decomposition (SVD) probably serves as a prevailing way to construct the capacity-achieving precoder for a single-user scenario by diagonalizing the MIMO channel matrix. To avoid introducing rather complicated bit loading strategies at the transmit side, the precoding scheme in [2] using geometric mean decomposition (GMD) $[3,4]$

\footnotetext{
*Correspondence: xdxu@ustc.edu.cn

Department of Electronic Engineering and Information Science, University of Science and Technology of China, Hefei, Anhui 230027, People's Republic of
} China and later the unitary channel decomposition (UCD) scheme [5] and the root-mean-square decomposition (RMSD) scheme [6] are successively proposed to achieve optimum bit error rate (BER) and channel throughput simultaneously.

Another attractive precoding scheme is recently introduced in [7] and [8] for MIMO multicast channels. Relying on the so-called joint equi-diagonal triangularization (JET) or, equivalently, the joint unitary triangularization (JUT) with fixed diagonal ratio, the research effort therein focuses primarily on constructing one common unitary precoding matrix for two users by jointly triangularizing their channel matrices. However, as one can see, the JET scheme usually results in subchannels with vastly different signal-to-noise ratios (SNRs) for every user, and the BER performance is substantially dominated by those who have the smallest gain on the diagonal, provided that the same modulation and coding scheme is used for all subchannels. Intuitively, conjecture that if there exists a joint GMD (JGMD) decomposition of two matrices, then each user can obtain multiple identical subchannels with equal

\section{是 Springer}

(c) 2014 Xu et al: licensee Springer. This is an Open Access article distributed under the terms of the Creative Commons Attribution License (http://creativecommons.org/licenses/by/2.0), which permits unrestricted use, distribution, and reproduction in any medium, provided the original work is properly cited. 
SNRs and the problem arising from JET can be eliminated thereafter. However, the existence condition of such a decomposition is available only for two $2 \times 2$ real-valued matrices [9]. To the authors' best knowledge, the existence condition of JGMD is still unknown for more generalized cases.

In this paper, we aim to alleviate the gain spread of the subchannels for each user and improve the SNR of the worst subchannels under the assumption that the same constellations are employed over all the subchannels by ignoring bit loading. Inspired by the successful application of GMD for multiuser MIMO broadcast scenarios [10], we would like to propose an improved block diagonal (BD) precoding scheme for a MIMO multicast channel with two users based on the hybrid usage of JET and JGMD, which can obtain better BER performance than the JET scheme counterpart. Given a prescribed dimension for every block, the idea behind the proposed precoding scheme lies in that for each submatrix of the block-wise unitary precoder, it can be selectively constructed by JET or JGMD according to particular channel realization. The main contributions are listed in the following:

1. The application of JGMD is considered, and the sufficient and necessary condition of the existence of JGMD is derived for the case of two complex-valued matrices with two columns. The analytical result reveals the connection with two-antenna channel realization so that JGMD can be conditionally used to perform submatrix construction for the proposed precoding scheme with more than two transmit antennas.

2. We reformulate and elaborately implement the BD precoding scheme by using JET as the unique submatrix construction algorithm, namely the pure BD-JET scheme in this paper, which can be seen as a supplemental work to [8]. New interesting insight into the property of diagonal elements is also shown for the upper triangular matrices.

3. Since JET may introduce large SNRs spread across different transmitted data streams and users, the hybrid usage of JET and JGMD is proposed to construct submatrices in the inner process of the precoding scheme. The existence condition of JGMD is used to determine whether JGMD is employed or not. The computational burden of the proposed precoding scheme is briefly discussed and compared with the JET scheme counterpart. Benefiting from the high probability of using JGMD instead of JET, the proposed scheme has the capability to guarantee a satisfactory BER performance for each user.

The remainder of this paper is structured as follows. Section 2 briefly describes the MIMO multicast channel model. In the next section, the existence of JGMD is studied and the proposed scheme is presented based on the combination of JET and JGMD. Section 4 illustrates several numerical examples to validate the advantage of the proposed scheme over the JET scheme. Finally, Section 5 summarizes this paper.

\section{Physical-layer multicasting system}

Consider a MIMO wireless physical-layer multicasting system consisting of one base station and two users. The base station is equipped with $M$ transmit antennas, and the $i$ th user is equipped with $N_{i} \geq M, i=1,2$, receive antennas. Assume that linear precoding is employed at the base station with a unitary precoding matrix $\mathbf{P} \in \mathbb{C}^{M \times M}$, and a common message $\mathbf{s} \in \mathbb{C}^{M}$ will be transmitted through the multicast channel. At the receive side, the $i$ th user receives

$$
\mathbf{y}_{i}=\mathbf{H}_{i} \mathbf{P s}+\mathbf{z}_{i}
$$

where $\mathbf{H}_{i} \in \mathbb{C}^{N_{i} \times M}$ is the channel matrix with full column rank for the $i$ th user and $\mathbf{z}_{i} \in \mathbb{C}^{N_{i}}$ is the circularly symmetric Gaussian white noise vector at the $i$ th user.

If the base station entirely knows in advance the instantaneous channel state, i.e., the full knowledge of matrix sequence $\left\{\mathbf{H}_{i}\right\}_{i=1}^{2}$, JET is applicable to construct the unitary precoder $\mathbf{P}$ by jointly decomposing $\left\{\mathbf{H}_{i}\right\}$ as follows:

$$
\mathbf{H}_{i}=a_{i} \mathbf{U}_{i} \mathbf{D}_{i} \mathbf{P}^{\dagger}, \quad i=1,2
$$

with $a_{i}=\sqrt[M]{\prod_{j=1}^{M} \sigma_{i, j}}$, where the sequence $\left\{\sigma_{i, j}\right\}_{j=1}^{M}$ is the singular value of $\mathbf{H}_{i}$. $\mathbf{U}_{1}$ and $\mathbf{U}_{2}$ are unitary matrices of dimensions $N_{1} \times N_{1}$ and $N_{2} \times N_{2}$, respectively, $\mathbf{D}_{1}$ and $\mathbf{D}_{2}$ are generalized upper triangular matrices with equal diagonal elements, and $(\cdot)^{\dagger}$ denotes the conjugate transpose.

Suppose a zero-forcing VBLAST (ZF-VBLAST) detector [11] is performed at the receive side, hence the $i$ th user yields a nulling output by multiplying $\mathbf{U}_{i}^{\dagger}$ to both sides of (1), i.e.,

$$
\hat{\mathbf{y}}_{i}=\mathbf{U}_{i}^{\dagger} \mathbf{y}_{i}=a_{i} \mathbf{D}_{i} \mathbf{s}+\hat{\mathbf{z}}_{i},
$$

where $\hat{\mathbf{z}}_{i}=\mathbf{U}_{i}^{\dagger} \mathbf{z}_{i}$. Ignoring the error propagation effect, a sequential signal detector using successive interference cancellation (SIC) will get $M$ parallel scalar subchannels, which can be expressed as

$$
\hat{y}_{i, j}=a_{i} d_{i, j j} s_{j}+\hat{z}_{i, j}, \quad j=1,2, \ldots, M,
$$

where $\hat{y}_{i, j}, s_{j}$, and $\hat{z}_{i, j}$ represent the $j$ th entry of the vectors $\hat{\mathbf{y}}_{i}, \mathbf{s}$, and $\hat{\mathbf{z}}_{i}$, respectively, and $d_{i, j j}$ denotes the $j$ th diagonal entry of the upper triangular matrix $\mathbf{D}_{i}$. Assume that the same modulation constellation is used in all the subchannels to reduce the system complexity, which is consistent with the HIPERLAN/2 and IEEE 802.11 standards. As a result, the overall BER performance of the 
JET scheme will be restricted by the subchannels with the smallest gain.

Notice that the JET precoding scheme explicitly adopts a promising unitary triangularization decomposition approach by (2) to jointly triangularize two matrices with one common unitary matrix. However, large spread of the diagonal elements $\left\{d_{i, j j}\right\}_{j=1}^{M}$ can be easily observed even for ordinary channel conditions, which will inevitably lead to the BER performance attenuation. Based on this observation, the motivation of this paper is to develop such a joint triangularization decomposition approach to dramatically reduce the spread or, equivalently, enlarge the smallest diagonal elements. Khina et al. [9] investigated this situation, but unfortunately, the sufficient and necessary condition of JGMD is still unknown not only for the case of more than two users but also for the case of more than two transmit antennas, except for the special case of two real-valued $2 \times 2$ matrices.

\section{The proposed precoding scheme}

To meet the requirement of high-speed data transmission, practical multicasting systems are usually expected to involve a large number of transmit antennas at the base station. Therefore, the equivalent channel matrices often contain columns larger than two in general. In particular, when the multicasting system encounters MIMO frequency-selective fading channels for all users, the dimensions of the equivalent channel matrices will dramatically increase, which will cause an inefficient use of JET at least for two aspects [12]: (1) the multiplication complexity equals $\mathcal{O}\left(M^{2}\right)$ while directly using a full-size unitary precoding matrix, which will increase with the increase of $M$, and (2) the requirement of feedback bits from the receiver will also increase with the increase of $M$ for encoding the precoding matrix.

In the sequel, we construct the block diagonal precoder for multicast channels with column dimensions larger than two. In this way, the aforementioned drawbacks will be mitigated to a great extent. Moreover, via matrix segmentation, it is possible to employ JGMD for reduceddimensional submatrices to achieve better performance. But before that, let us first consider the existence of JGMD in the next subsection.

\subsection{JGMD for complex-valued matrix case}

For the case of $M=2$, without loss of generality, denote the unitary matrix $\mathbf{P}$ as

$$
\mathbf{P}=\left[\mathbf{p}_{1}, \mathbf{p}_{2}\right]=\left[\begin{array}{cc}
\cos \theta & -\sin \theta e^{-j \psi} \\
\sin \theta e^{-j \psi} & \cos \theta
\end{array}\right]
$$

where $\mathbf{p}_{i}$ is the $i$ th column vector of $\mathbf{P}$ and $\theta \in[0, \pi / 2)$, $\psi \in[0,2 \pi)$. Then the following lemma states the existence condition of JGMD for two complex-valued matrices.
Lemma 1. Let $\mathbf{e}=\left[\sigma_{1,1} \sigma_{1,2}, \sigma_{2,1} \sigma_{2,2}\right]^{T}$ be the vector whose elements are products of the singular values of $\left\{\mathbf{H}_{i}\right\}_{i=1}^{2}$. There exists JGMD for $\left\{\mathbf{H}_{i}\right\}_{i=1}^{2}$ if and only if the following equation:

$$
\mathbf{c}+\mathbf{G x}=\mathbf{e}
$$

has solutions for real-valued vector $\mathbf{x}$, where $\mathbf{x} \triangleq$ $[\cos 2 \theta, \sin 2 \theta \cos \psi, \sin 2 \theta \sin \psi]^{T}, \mathbf{c}=\left[\frac{\left\|\mathbf{H}_{1}\right\|_{F}^{2}}{2}, \frac{\left\|\mathbf{H}_{2}\right\|_{F}^{2}}{2}\right]^{T}$, $\|\cdot\|_{F}$ stands for the Frobenius norm, and

$$
\mathbf{G}=\left[\begin{array}{lll}
\frac{h_{1,11}-h_{1,22}}{2} & \Re\left\{h_{1,12}\right\} & -\Im\left\{h_{1,12}\right\} \\
\frac{h_{2,11}-h_{2,22}}{2} & \Re\left\{h_{2,12}\right\} & -\Im\left\{h_{2,12}\right\}
\end{array}\right] \in \mathbb{R}^{2 \times 3}
$$

with $h_{i, j k}$ represented as the entry of the jth row and $k t h$ column of $\mathbf{H}_{i}^{\dagger} \mathbf{H}_{i}$.

Proof. For any unitary $\mathbf{P}$, one can obtain the QR decomposition of $\mathbf{H}_{i} \mathbf{P}$ as follows:

$$
\mathbf{H}_{i} \mathbf{P}=\mathbf{Q}_{i} \mathbf{R}_{i}, \quad i=1,2 .
$$

In view of (5), the first column of both sides of (8) becomes $\mathbf{H}_{i} \mathbf{p}_{1}=\mathbf{Q}_{i}\left[r_{i, 11}, 0\right]^{T}$ with $r_{i, 11}$ denoting the first diagonal element of $\mathbf{R}_{i}$. Subsequently, the square form of $r_{i, 11}$ is obtained by

$$
r_{i, 11}^{2}=\mathbf{p}_{1}^{\dagger} \mathbf{H}_{i}^{\dagger} \mathbf{H}_{i} \mathbf{p}_{1}, \quad i=1,2 .
$$

Resorting to the statement of Lemma 1 in [13], the righthand side of (9) can be transformed into the matrix form of $\mathbf{c}+\mathbf{G x}$, while the left-hand side of (9) essentially represents the SNR of the first subchannel for the $i$ th user. Therefore, if there exists JGMD for matrix sequence $\left\{\mathbf{H}_{i}\right\}_{i=1}^{2}$, then both $r_{i, 11}^{2}$ and $r_{i, 22}^{2}$ should be equal to $\sigma_{i, 1} \sigma_{i, 2}$ from the determinant viewpoint, which means that (6) has solutions, and vice versa.

Remark 1. Lemma 1 reveals the relationship between the existence condition of JGMD and the solution of a constraint equation for two complex-valued matrices. It is straightforward from this lemma that, if existing, the resulting solution of (6) has the form of

$$
\mathbf{x}=\mathbf{G}^{\ddagger}(\mathbf{e}-\mathbf{c})+\rho \mathbf{g},
$$

where $\mathbf{g}$ is chosen from the unit-length basis vectors in the null space of $\mathbf{G}, \rho$ is an arbitrary real value to meet the norm constraint of $\|\mathbf{x}\|=1$, and (.) denotes pseudoinverse. Apparently, the sufficient and necessary condition that Lemma 1 tells is equivalent to the following one:

$$
\left\|\mathbf{G}^{\ddagger}(\mathbf{e}-\mathbf{c})\right\| \leq 1 \text {. }
$$

When the solution $\mathbf{x}$ is obtained, the corresponding angular parameters $\theta$ and $\psi$ can then be uniquely acquired through the definition. In this case, the unitary matrix $\mathbf{P}$ is obtained finally. 
Remark 2. Consider more general cases of $K>2$ users in one multicasting system with an extension version of $\mathbf{c} \in \mathbb{R}^{K}$, $\mathbf{e} \in \mathbb{R}^{K}$, and $\mathbf{G} \in \mathbb{R}^{K \times 3}$, respectively. It is clear from (6) that no proper solution $\mathbf{x} \in \mathbb{R}^{3}$ will exist with probability 1 . Therefore, we only focus on precoding for multicasting systems with two users. For a complex-valued matrix with column dimension $M>2$, the existence condition of JGMD is still an open issue.

\subsection{BD-JET decomposition with discussions about diagonal elements}

With the merit of Lemma 1, we are now dedicated in jointly decomposing two matrices into upper triangular forms with equal elements on each diagonal. Before proceeding with the design procedure, we are willing to reformulate BD-JET carefully with full implementation for reasons of completeness, which is not explicitly described in [8]. The following lemma indicates the principle of this decomposition approach.

Lemma 2. Full and equal column rank matrices $\left\{\mathbf{H}_{i}\right\}_{i=1}^{2}$ can be jointly triangularized into $B$ blocks as follows:

$$
\mathbf{H}_{i}=\mathbf{Q}_{i} \mathbf{T}_{i} \mathbf{P}^{\dagger}, \quad i=1,2,
$$

where $\mathbf{Q}_{i}$ is an $N_{i} \times M$ semi-unitary matrix, $\mathbf{P}$ is an $M \times$ $M$ block diagonal matrix of the form $\operatorname{diag}\left(\mathbf{P}_{1}, \mathbf{P}_{2}, \ldots, \mathbf{P}_{\mathrm{B}}\right)$, where each individual block $\mathbf{P}_{j}$ is a $b_{j} \times b_{j}$ unitary matrix and $\sum_{j=1}^{B} b_{j}=M .\left\{\mathbf{T}_{i}\right\}_{i=1}^{2}$ are $M \times M$ upper triangular matrices with equal diagonal ratio for each block.

Proof. Before starting the proof process, we are concerned with an equivalent structure of the regular-form JET via (2), which is referred to as an alternative-form JET in this paper. In other words, adopting JET to $\left\{\mathbf{H}_{i}\right\}_{i=1}^{2}$ will result in the following two equivalent forms:

$$
\mathbf{H}_{i}=a_{i} \underbrace{\left[\mathbf{U}_{i, 1}, \mathbf{U}_{i, r}\right.}_{\mathbf{U}_{i, \mathrm{ET}}} \underbrace{\left[\begin{array}{c}
\mathbf{D}_{i, 1} \\
\mathbf{0}
\end{array}\right]}_{\mathbf{D}_{i, \mathrm{JET}}} \mathbf{V}_{\mathrm{JET}}^{\dagger}=a_{i} \mathbf{U}_{i, 1} \mathbf{D}_{i, 1} \mathbf{V}_{\mathrm{JET}}^{\dagger}
$$

by appropriate rearrangement, where $\mathbf{U}_{i, r}$ is an $N_{i} \times\left(N_{i}-\right.$ $M)$ matrix that contains the last $N_{i}-M$ columns of $\mathbf{U}_{i, \mathrm{JET}}$, and $\mathbf{U}_{i, 1}, \mathbf{D}_{i, 1}$ have different dimensions of $N_{i} \times M$ and $M \times M$ from those of $\mathbf{U}_{i, \text { JET }}$ and $\mathbf{D}_{i, \text { JET }}$, respectively. For clarification, the arguments with 'JET' suffix denote that they are generated by the regular-form JET.

Relying on the principle of the BD-GMD algorithm [10], we can segment $\left\{\mathbf{H}_{i}\right\}_{i=1}^{2}$ to acquire

$$
\mathbf{H}_{i}=\left[\mathbf{H}_{i, 1}, \mathbf{H}_{i, r}\right]=\left[\mathbf{Q}_{i, 1}, \mathbf{Q}_{i, r}\right]\left[\begin{array}{cc}
\mathbf{T}_{i, 1} & \mathbf{E}_{i, r} \\
\mathbf{0} & \mathbf{T}_{i, r}
\end{array}\right]\left[\begin{array}{cc}
\mathbf{P}_{1} & \mathbf{0} \\
\mathbf{0} & \mathbf{P}_{r}
\end{array}\right]^{\dagger}
$$

with $\mathbf{H}_{i, 1}=\mathbf{Q}_{i, 1} \mathbf{T}_{i, 1} \mathbf{P}_{1}^{\dagger}$, where for any matrix $\mathbf{X}_{i}, \mathbf{X}_{i, 1}$ contains the first $b_{1}$ columns of $\mathbf{X}_{i}$, while $\mathbf{X}_{i, r}$ contains the rest of the columns. For the first block, the alternative-form JET via (13) can be used to manipulate $\left\{\mathbf{H}_{i, 1}\right\}_{i=1}^{2}$ as

$$
\mathbf{H}_{i, 1}=\alpha_{i, 1} \mathbf{U}_{i, 1} \mathbf{D}_{i, 1} \mathbf{V}_{1, \mathrm{JET}}^{\dagger} .
$$

From (15), it is reasonable to obtain the exact solution of $\mathbf{P}_{1}, \mathbf{T}_{i, 1}$, and $\mathbf{Q}_{i, 1}$ by setting $\mathbf{P}_{1}=\mathbf{V}_{1, \mathrm{JET}}, \mathbf{T}_{i, 1}=\alpha_{i, 1} \mathbf{D}_{i, 1}$, and $\mathbf{Q}_{i, 1}=\mathbf{U}_{i, 1}$, respectively. And the diagonal ratio between $\mathbf{T}_{1,1}$ and $\mathbf{T}_{2,1}$ is fixed by $\frac{\alpha_{1,1}}{\alpha_{2,1}}$.

Due to the orthogonalization between $\mathbf{Q}_{i, 1}$ and $\mathbf{Q}_{i, r}$, the remainder of $\mathbf{H}_{i}$ satisfies that

$$
\left(\mathbf{I}-\mathbf{Q}_{i, 1}^{\dagger} \mathbf{Q}_{i, 1}\right) \mathbf{H}_{i, r}=\mathbf{Q}_{i, r} \mathbf{T}_{i, r} \mathbf{P}_{r}^{\dagger},
$$

which has the same decomposition pattern as that of $\mathbf{H}_{i, 1}$. Thus, we can continue the above operation recursively until all the $B$ blocks have been factorized. Consequently, $\mathbf{Q}_{i}, \mathbf{T}_{i}$, and $\mathbf{P}$ consist of the output submatrices from every iteration, and finally, (12) is reached.

Remark 3. Lemma 2 allows for a block diagonal decomposition approach, named as BD-JET, to triangularize two matrices simultaneously. The proof is constructive and results in a detailed decomposition procedure, which is not pointed out in [8]. BD-JET can also be seen as a relaxed application of JET since JET is employed in the inner iteration process of BD-JET. Moreover, if there is only one block instead, i.e., $B=1$, then BD-JET will be reduced to JET. And if $B=M$, as a result, the unitary matrix $\mathbf{P}$ will degrade to an identity matrix. However, there are at least two evidences that state the differences between BD-JET and JET. The first one is that in JET mode and in BD-JET mode, the unitary matrix $\mathbf{P}$ may have a different structure, except for the same matrix dimension. BD-JET possesses a block diagonal matrix $\mathbf{P}$ which contains several unitary submatrices. The second one lies in that in JET mode, the ratio of the diagonal elements of $\mathbf{D}_{i}$ is fixed; nevertheless, in BD-JET mode, the ratio of the diagonal elements of $\mathbf{T}_{i}$, as mentioned in the next remark, is block-equal and not identical between blocks in general.

Let $\mathbf{H}_{i, k}=\mathbf{Q}_{i, k} \mathbf{T}_{i, k} \mathbf{P}_{k}^{\dagger}$ denote the BD-JET results of the first $\sum_{j=1}^{k} b_{j}$ columns of matrices $\left\{\mathbf{H}_{i}\right\}_{i=1}^{2}$. The following corollary describes the property of the $k$ th block diagonal elements of $\mathbf{T}_{i, k}$, defined as $\left[t_{i, k, 1}, t_{i, k, 2}, \ldots, t_{i, k, b_{k}}\right]$.

Corollary 1. The kth block diagonal entries of the upper triangular matrices $\left\{\mathbf{T}_{i, k}\right\}_{i=1}^{2}$ have the following property:

$$
\prod_{j=1}^{b_{k}} t_{i, k, j}=\left\{\begin{array}{ll}
\sqrt{\frac{a_{i, k}}{a_{i, k-1}}} & \text { for } k>1 \\
\sqrt{a_{i, k}} & \text { for } k=1
\end{array},\right.
$$

where $a_{i, k}=\operatorname{det}\left(\mathbf{H}_{i, k}^{\dagger} \mathbf{H}_{i, k}\right)$. 
Proof. For the first block, i.e., $k=1$, it is apparent that $a_{i, 1}=\prod_{j=1}^{b_{1}} t_{i, 1, j}^{2}$. For the case of $k>1$, according to $\mathbf{H}_{i, k}=$ $\mathbf{Q}_{i, k} \mathbf{T}_{i, k} \mathbf{P}_{k}^{\dagger}$, it holds that

$$
a_{i, k}=\operatorname{det}\left(\mathbf{H}_{i, k}^{\dagger} \mathbf{H}_{i, k}\right)=\operatorname{det}\left(\mathbf{T}_{i, k}^{\dagger} \mathbf{T}_{i, k}\right)=\prod_{j=1}^{k} \prod_{l=1}^{b_{j}} t_{i, j, l}^{2}
$$

As a consequence, the connection between the determinants of adjacent blocks reads

$$
a_{i, k}=a_{i, k-1} \prod_{j=1}^{b_{k}} t_{i, k, j}^{2}
$$

In other words, the product of the $k$ th block diagonal entries of the upper triangular matrices $\mathbf{T}_{i, k}$ is characterized by (17).

Remark 4. From Corollary 1, the ratio of the $k$ th block elements between the upper triangular matrices $\left\{\mathbf{T}_{i}\right\}$ holds for $r_{k}=\sqrt[2 b_{k}]{\frac{a_{1, k} a_{2, k-1}}{a_{1, k-1} a_{2, k}}}$. In fact, $r_{k}$ may be controlled inherently by determinants of the current block as well as the former one. As for JET, the ratio would be kept as $r_{\mathrm{JET}}=\sqrt[2 M]{\frac{a_{1, B}}{a_{2, B}}}$ for the whole diagonal elements. For the case of $B=1, r_{1}=r_{\text {JET }}$ holds true intuitively. However, as $B$ increases, this relationship is invalid in general except that $\left\{\mathbf{H}_{i}\right\}$ have special matrix structures. In addition, we note that the ratio $r_{k}$ majorizes $r_{\text {JET }}$ by majorization theory [14], and it is proved by the numerical evaluations in the next section that $\mathbf{T}_{i}$ has relatively smaller gain spread in the diagonal than $\mathbf{D}_{i}$ with high probability, which will lead to an improved smallest gain for the worst subchannels.

Assuming $\hat{\mathbf{H}}_{i, k}$ consists of the last $b_{k}$ columns of $\mathbf{H}_{i, k}$, we can use the properties of the block matrix determinant to further get the following relationship:

$$
a_{i, k}=a_{i, k-1} \operatorname{det}\left(\mathbf{W}_{i, k}\right)
$$

where $\mathbf{W}_{i, k}=\hat{\mathbf{H}}_{i, k}^{\dagger} \hat{\mathbf{H}}_{i, k}-\hat{\mathbf{H}}_{i, k}^{\dagger} \mathbf{H}_{i, k-1}\left(\mathbf{H}_{i, k-1}^{\dagger} \mathbf{H}_{i, k-1}\right)^{-1}$ $\mathbf{H}_{i, k-1}^{\dagger} \hat{\mathbf{H}}_{i, k}$.

\subsection{The proposed scheme}

Although the BD-JET decomposition has no specific constraint on the size of submatrices, the use of JET may still produce large gain spread across different subchannels in the decomposition procedure. In this subsection, we put forward an improved block diagonal precoding scheme for multicasting system with two users, where the unitary precoding matrix is constructed based on a combination of JET and JGMD. Actually, JGMD will act as the major decomposition approach for every two submatrices, thanks to the high existence probability, which is validated by numerical evaluations in the next section. Algorithm 1 summarizes the details of the proposed scheme.

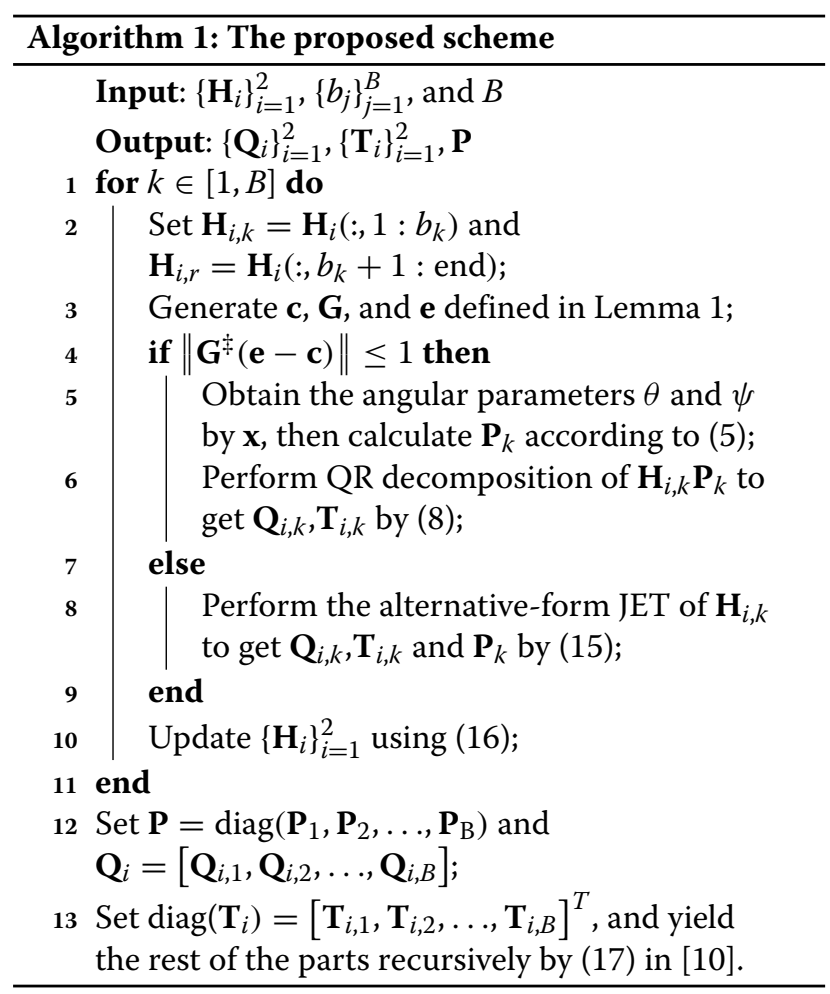

Remark 5. Bearing in mind that the existence of JGMD is questionable for higher dimensions (i.e., for more than two transmit antennas), all the variables $\left\{b_{j}\right\}_{j=1}^{B}$ should have equal values of two for matrices $\left\{\mathbf{H}_{i}\right\}_{i=1}^{2}$ who have even number of columns. For the case of odd number of columns in $\left\{\mathbf{H}_{i}\right\}_{i=1}^{2}$, all the variables $\left\{b_{j}\right\}$ still have values of two, but the last one where $b_{B}=M-\sum_{j=1}^{B-1} b_{j}$ is guaranteed with $B=\left\lceil\frac{M-1}{2}\right\rceil$. And JET is automatically assigned for the joint triangularization decomposition of the last block in the current situation, which is omitted in Algorithm 1 for concise clarification purposes. Besides, in contrast with the proposed scheme, we rename the aforementioned BD-JET scheme including JET as the unique algorithm for submatrix construction as the pure BD-JET scheme hereafter.

Remark 6. For simplicity, let $N_{1}=N_{2}=N$ and each block has equal size, i.e., $b_{j}=L, \forall j$. Then the computational complexity of the proposed scheme can be 
approximately figured out in terms of the number of flops as

$$
\begin{aligned}
f= & \mathcal{O}(M N L)+\mathcal{O}\left(M L^{2}\right)+\mathcal{O}\left(M N^{2}\right) \\
& +\mathcal{O}\left((M-L) \frac{2 N M^{2}+M N^{2}+N M L+L N^{2}}{2 L}\right) .
\end{aligned}
$$

In contrast, the number of flops for JET can be computed by

$$
f_{\mathrm{JET}}=\mathcal{O}\left(N M^{2}\right)+\mathcal{O}\left(M^{3}\right)
$$

for the same $\left\{\mathbf{H}_{i}\right\}_{i=1}^{2}$. As can be seen from (21) and (22), the computational complexity of the proposed scheme is somewhat larger than the JET counterpart since typically, $L<M$. Nevertheless, due to the bock diagonal decomposition, the resulting block diagonal precoder $\mathbf{P}$ can reduce the multiplication complexity from $\mathcal{O}\left(M^{2}\right)$ to $\mathcal{O}\left(\frac{M^{2}}{B}\right)$ in general.

\section{Simulation results}

In this section, several numerical examples are performed to demonstrate the efficiency of the proposed scheme. A single-group multicasting system with two users is employed and without loss of generality, the number of receive antennas for each user is set equal to each other, i.e., $N_{1}=N_{2}=N$. Transmit symbols are commonly chosen from quadrature phase shift keying (QPSK) constellation throughout this paper.

Example 1. For the proposed scheme, it is worth noting that the existence probability of JGMD seriously affects the gain spread along the diagonal of the upper triangular matrices. Too low probability will restrict the proposed scheme to have any advantage over the JET scheme. Theoretical analysis of this stochastic characteristics will become very cumbersome owing to the complicated probability model. For this reason, we start by first estimating the probability of interest by Monte Carlo trials. Assuming that $\left\{\mathbf{H}_{i}\right\}_{i=1}^{2}$ are of $N \times 2$ independent and identically distributed (i.i.d) Rayleigh fading channels, Table 1 establishes the existence probability of JGMD versus $N$, where it is clear that JGMD exists in most cases, especially when $N$ has large values.

Given $\left\{\mathbf{H}_{i}\right\}_{i=1}^{2}$ with $N=M$, Table 2 shows the possibility that whether or not the smallest diagonal elements

Table 1 Probability of the existence of JGMD

\begin{tabular}{cccccccc}
\hline & \multicolumn{8}{c}{$\boldsymbol{N}$} \\
\cline { 2 - 8 } & $\mathbf{2}$ & $\mathbf{3}$ & $\mathbf{4}$ & $\mathbf{5}$ & $\mathbf{6}$ & $\mathbf{7}$ & $\mathbf{8}$ \\
\hline Probability & 0.695 & 0.829 & 0.881 & 0.907 & 0.928 & 0.936 & 0.949
\end{tabular}

Table 2 Probability of improved smallest gain for MIMO frequency-flat channels

\begin{tabular}{lccccc}
\hline & \multicolumn{5}{c}{$\boldsymbol{N}=\boldsymbol{M}$} \\
\cline { 2 - 6 } & $\mathbf{4}$ & $\mathbf{5}$ & $\mathbf{6}$ & $\mathbf{7}$ & $\mathbf{8}$ \\
\hline Probability & 0.861 & 0.861 & 0.862 & 0.862 & 0.863 \\
\hline
\end{tabular}

caused by the proposed scheme are greater than the pure BD-JET case. From this table, it appears that the proposed scheme always has high probability to produce subchannels with better SNRs than the pure BD-JET. Since JGMD is essentially unused for the case of $N=M \leq 3$, which means that the proposed scheme is equivalent to the pure $\mathrm{BD}-J E T$ one in this case, therefore we do not consider this situation in this table. Assuming $N=M$, more evidences can be observed in Table 3, where the multicasting system suffers from MIMO frequency-selective channels with $\mu$ fading paths.

Example 2. In this example, the proposed scheme is investigated from the BER performance aspect for multicasting in MIMO frequency-flat fading channels, where the system parameters are set as $M=N=8, B=4$, and $b_{j}=2, \forall j$. Figure 1 depicts the comparative curves of average BER performance of each user versus SNR. As can be seen from this figure, each user almost has the same BER property, and the proposed scheme achieves superior performance over the JET scheme and the pure $\mathrm{BD}-J E T$ scheme in moderate to high SNR regimes. Due to the unitary precoding, the proposed scheme is expected to have the same throughput as that of the JET scheme for the multicasting system. However, the smallest gain of the worst subchannels is improved by the proposed scheme at the cost of a small increase in computational complexity. The resulting performance gap implies the effective application of JGMD as the major decomposition approach for the inner process of the proposed scheme.

Notice that the unitary precoder $\mathbf{P}$ consists of four $2 \times 2$ unitary submatrices in this case. Therefore, it is clear that the block diagonal structure of $\mathbf{P}$ can reduce matrix multiplication complexity from $\mathcal{O}\left(M^{2}\right)$ to $\mathcal{O}\left(\frac{M^{2}}{4}\right)$ in comparison with the JET scheme.

Table 3 Probability of improved smallest gain for frequency-selective MIMO channels

\begin{tabular}{cccccccc}
\hline & \multicolumn{7}{c}{$\boldsymbol{\mu}$} \\
\cline { 2 - 8 } & $\mathbf{2}$ & $\mathbf{3}$ & $\mathbf{4}$ & $\mathbf{5}$ & $\mathbf{6}$ & $\mathbf{7}$ & $\mathbf{8}$ \\
\hline Probability & 0.973 & 0.975 & 0.977 & 0.981 & 0.984 & 0.986 & 0.987
\end{tabular}




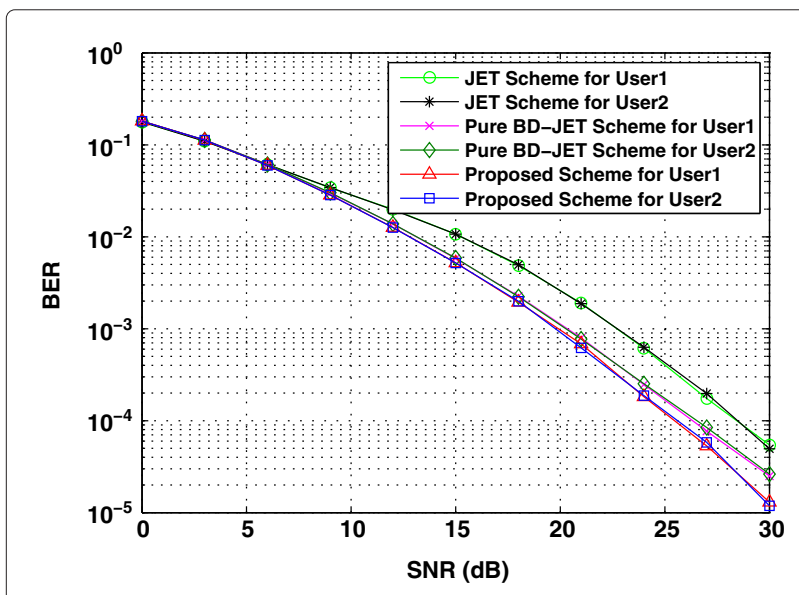

Figure 1 BER performance versus SNR for MIMO frequency-flat channel.

Example 3. Another favorite multicast scenario is about the block transmission through MIMO frequencyselective fading channels with $\mu$ distinguishable paths. In this example, the system uses a zero-padded transmission mechanism, which means that the system will transmit $\mu$ zero vectors after $\tau$ symbol vectors in every $\tau+\mu$ symbol duration. In fact, we can acquire the $(\tau+\mu) N \times \tau M$ equivalent channel matrix $\left\{\mathbf{H}_{i}\right\}$ in block Toeplitz structure in the current situation.

In the sequel, Figure 2 illustrates the trajectories of the BER performance versus SNR for two users with $M=$ $N=2, \mu=4$ effective paths, and $\tau=4$ symbol block size. In this case, the equivalent channel matrix actually has a dimension of $16 \times 8$. Similar behaviors manifest that the proposed scheme has reliable performance better

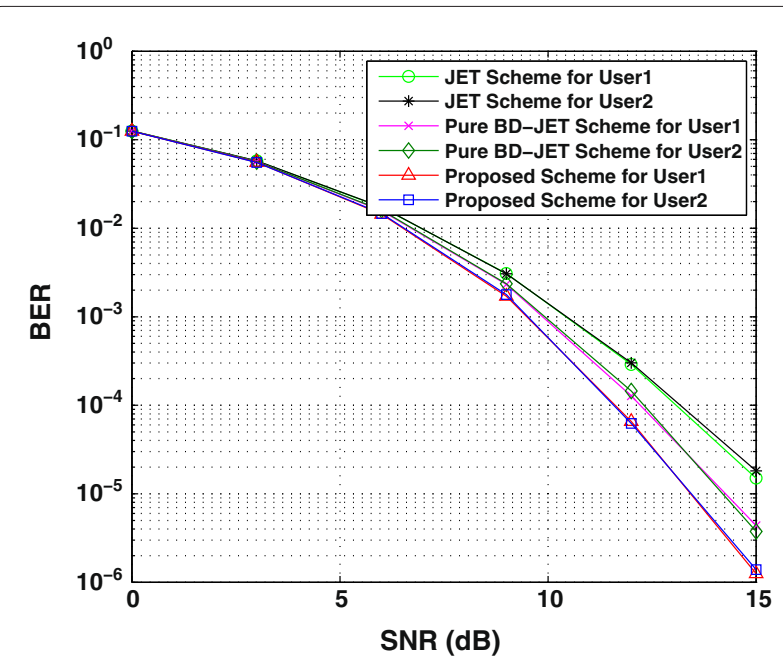

Figure 2 BER performance versus SNR for frequency-selective MIMO channel. than the JET scheme in moderate to high SNR regimes. The pure BD-JET scheme is also examined and plotted in this figure. It is shown that in contrast to the pure BD-JET scheme, the proposed one can still yield a performance improvement about $1 \mathrm{~dB}$ in the high SNR case. The BER performance versus the symbol block size $\tau$ is also shown in Figure 3 at $\mathrm{SNR}=15 \mathrm{~dB}$. It is clear from this figure that although the bandwidth efficiency increases as $\tau$ increases, the BER performance degrades a lot for the proposed scheme as well as for the JET scheme. Similarly, performance properties have also been mentioned for the BD-GMD scheme in [12] for unicast scenarios.

\section{Conclusions}

In this paper, we present an improved block diagonal precoding scheme to construct a unitary precoder for a MIMO multicast channel with two users by using joint triangularization decomposition. An elaborate implementation of the pure BD-JET scheme is provided by means of JET. Although the pure BD-JET scheme achieves relative advantages without an explicit constraint on the size of submatrices, the use of JET may suffer from large SNR spread across different transmit data streams and users. Fully aware of the existence condition of JGMD for two transmit antennas, we introduce the hybrid application model of JGMD and JET to construct submatrices in the inner process of the precoding scheme. The high existence probability of JGMD is proved by numerical results so that in the proposed scheme, JGMD is observed to be the major decomposition algorithm for submatrix construction, as compared with the JET counterpart. Besides the analytical result of the computational complexity, properties of the diagonal elements are also derived to gather useful insights into the proposed scheme. Several numerical examples demonstrate that the proposed scheme can guarantee satisfactory BER performance over the JET

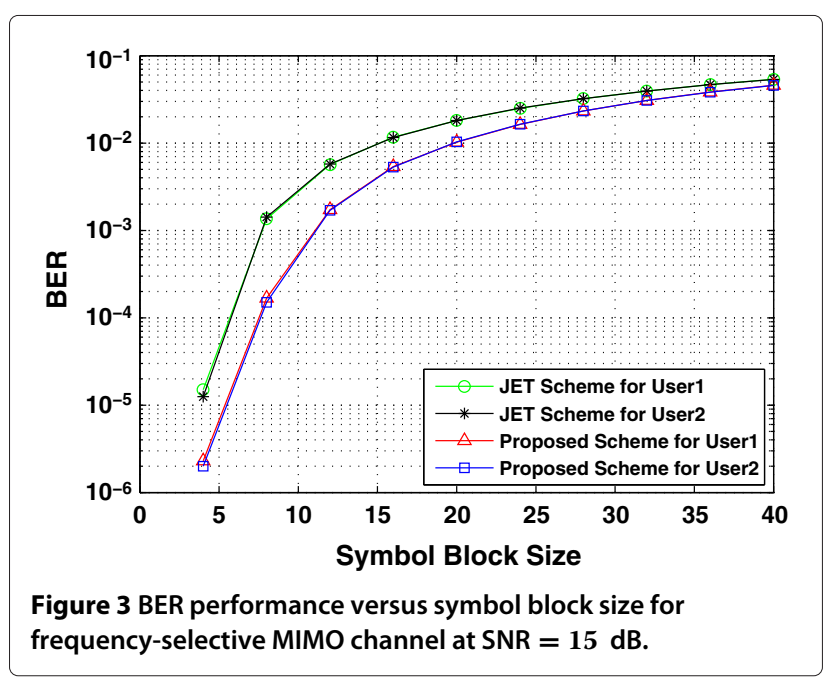


scheme in moderate to high SNR regimes, especially when the MIMO multicast system is equipped with large-scale antennas.

\section{Competing interests}

The authors declare that they have no competing interests.

\section{Acknowledgements}

This work was supported by the National Natural Science Foundation of China under grant number 61271272, the National Science and Technology Special Projects of China under grant number 2012ZX03001007-002, and the National High Technology Research and Development Program of China (863 Program) under grant number 2012AA01A502. The authors would like to acknowledge Prof. Xuchu Dai for his valuable comments and suggestions.

Received: 30 July 2013 Accepted: 18 March 2014

Published: 26 March 2014

\section{References}

1. M Vu, A Paulraj, MIMO wireless linear precoding. IEEE Signal Process. Mag. 24(5), 86-105 (2007)

2. Y Jiang, W Hager, J Li, The geometric mean decomposition. Linear Algebra Appl. 396, 373-384 (2005)

3. P Kosowski, A Smoktunowicz, On constructing unit triangular matrices with prescribed singular values. Computing. 64(3), 279-285 (2000)

4. J-K Zhang, A Kavcic, X Ma, KM Wong, Design of unitary precoders for ISI channels, in Proc. IEEE Intern. Conf. on Acous. Speech and Signal Processing, vol. 3 (Orlando, FL, USA, 13-17 May 2002), pp. 2265-2268

5. Y Jiang, W Hager, J Li, Uniform channel decomposition for MIMO communications. IEEE Trans. Signal Process. 53(11), 4283-4294 (2005)

6. T Hwang, Y Kwon, Root mean square decomposition for EST-based spatial multiplexing systems. IEEE Trans. Signal Process. 60(3), 1295-1306 (2012)

7. A Khina, Y Kochman, U Erez, Decomposing the MIMO broadcast channel, in Proc. 48th Annual Allerton Conf. on Commun., Control and Computing (Allerton, IL, USA, 29 Sept-1 Oct 2010), pp.102-106

8. A Khina, Y Kochman, U Erez, Joint unitary triangularization for MIMO networks. IEEE Trans. Signal Process. 60(1), 326-336 (2012)

9. A Khina, A Hitron, $U$ Erez, Modulation for MIMO networks with several users, in Proc. IEEE International Symposium on Information Theory (St. Petersburg, Russia, 31 July-5 Aug 2011), pp. 2452-2456

10. S Lin, WL Ho, YC Liang, Block diagonal geometric mean decomposition (BD-GMD) for MIMO broadcast channels. IEEE Trans. Wireless Commun. 7(7), 2778-2789 (2008)

11. GJ Foschini, GD Golden, RA Valenzuela, PW Wolniansky, Simplified processing for high spectral efficiency wireless communication employing multiple-element arrays. Wireless Personal Commun 6, 311-335 (1999)

12. C-C Weng, PP Vaidyanathan, Block diagonal GMD for zero-padded MIMO frequency selective channels. IEEE Trans. Signal Process. 59(2), 713-727 (2011)

13. B Du, $Y$ Jiang, $X X u, X$ Dai, Transmit beamforming for MIMO multicast channels, in Proc. IEEE International Conference on Commun (Ottawa, Cannada, 10-15 June 2012), pp. 3800-3805

14. DP Palomar, Y Jiang, MIMO transceiver design via majorization theory. Found. Trends Commun. Inform. Theory 3, 331-551 (2006)

\section{doi:10.1186/1687-6180-2014-39}

Cite this article as: Xu et al:: An improved block diagonal precoding scheme for MIMO multicast channel with two users. EURASIP Journal on Advances in Signal Processing 2014 2014:39.

\section{Submit your manuscript to a SpringerOpen ${ }^{\mathcal{O}}$ journal and benefit from:}

- Convenient online submission

- Rigorous peer review

- Immediate publication on acceptance

- Open access: articles freely available online

- High visibility within the field

- Retaining the copyright to your article

Submit your next manuscript at $\gg$ springeropen.com 